\title{
SURGICAL TREATMENT OF GIANT-CELL TUMORS OF THE DISTAL EPIPHYSIS OF THE RADIUS
}

doi: $10.2478 /$ rojost-2018-0030

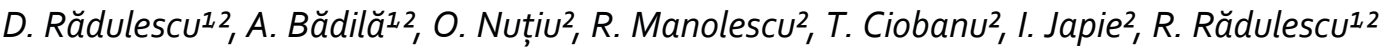 \\ "Carol Davila" University of Medicine and Pharmacy, Bucharest, Romania \\ ${ }^{2}$ Orthopaedics and Traumatology Department, University Emergency Hospital, Bucharest, Romania
}

Introduction. Giant-cell tumor of the bone is a benign tumor, but with high local aggressiveness, even with risk of remote metastasis.

Material and methods. We present the case of a 57-year-old woman, without significant pathological history, who, after clinical, imagistic and anatomopathological investigations, was diagnosed with giant cell tumor of the right distal radius. The patient underwent surgery and due to the size of the tumor and destruction of the surrounding cortical bone, segmental resection of the tumor in oncological limits was performed. The bone defect was filled with the proximal one third of the ipsilateral fibula, fixed to the remaining radius diaphysis with a plate and screws. Also, the autograft was stabilized to the proximal row of the carpal bones with 2 k-wires for 6 weeks. Postoperatively, clinical and X-ray check-ups were performed at 6, 12, 24 weeks and 1 year after surgery.

Results. According to Mayo functional assessment score, the results were good. At 1 year after surgery, the patient gained 85 points, representing a good functional outcome of the surgery. This way, the wrist joint mobility and the carpal cartilage were preserved, providing a barrier against distal migration of any remaining tumoral cells, as well.

Conclusions. It can be stated that in aggressive giant cell tumors located at the distal radius, the best therapeutic option is a segmental resection of the lesion followed by the replacement of the bone defect with a proximal fibular autograft. This method provides the best postoperative functional results with a lower risk of local recurrence and does not require microvascular surgery or access to a bone bank.

Keywords: giant cell tumor, segmental resection, fibular graft 